\title{
Corrigendum: Rapid generation of fully human monoclonal antibodies specific to a vaccinating antigen
}

Kenneth Smith, Lori Garman, Jens Wrammert, Nai-Ying Zheng, J Donald Capra, Rafi Ahmed \& Patrick C Wilson Nat. Protoc. 4, 372-384 (2009); doi:10.1038/nprot.2009.3; published online 26 February 2009; corrected after print 24 January 2014 In the version of this article initially published, the sequence of primer VH3a-sense in Table 1 incorrectly indicated a ' $\mathrm{C}$ ' at position 12 instead of a ' $G$. The error has been corrected in the HTML and PDF versions of the article.

\section{Erratum: Tethered particle analysis of supercoiled circular DNA using peptide nucleic acid handles}

Kamilla Norregaard, Magnus Andersson, Peter Eigil Nielsen, Stanley Brown \& Lene B Oddershede

Nat. Protoc. 9, 2206-2223 (2014); doi:10.1038/nprot.2014.152; published online 21 August 2014; corrected after print 12 September 2014

In the version of this article initially published, the source from which Figures 2, 4, 8 and 9 was adapted was not cited and credited correctly. The error has been corrected in the HTML and PDF versions of the article.

\section{Corrigendum: Bio-orthogonal labeling as a tool to visualize and identify newly synthesized proteins in Caenorhabditis elegans}

Milena Ullrich, Vanessa Liang, Yee Lian Chew, Samuel Banister, Xiaomin Song, Thiri Zaw, Hong Lam, Slavica Berber, Michael Kassiou, Hannah R Nicholas \& Jürgen Götz

Nat. Protoc. 9, 2237-2255 (2014); doi:10.1038/nprot.2014.150; published online 28 August 2014; corrected after print 12 September 2014

In the version of this article initially published, one of the two affiliations of one of the authors (Michael Kassiou) was incorrect. The mistaken affiliation read: "FFaculty of Health Sciences, Macquarie University, Sydney, New South Wales, Australia." The correct affiliation is: "Faculty of Health Sciences, University of Sydney, Sydney, New South Wales, Australia." The error has been corrected in the HTML and PDF versions of the article.

\section{Corrigendum: Detecting ultralow-frequency mutations by Duplex Sequencing}

Scott R Kennedy, Michael W Schmitt, Edward J Fox, Brendan F Kohrn, Jesse J Salk, Eun Hyun Ahn, Marc J Prindle, Kawai J Kuong, Jiang-Cheng Shen, Rosa-Ana Risques \& Lawrence A Loeb Nat. Protoc. 9, 2586-2606 (2014); doi:10.1038/nprot.2014.170; corrected online 22 October 2014

In the version of this article initially published online, the sequence for the MWS21 oligonucleotide was incorrectly listed in Table 3 as 5 -CCAGCAGAAGACGGCATACGAGATXXXXXXGTGACTGGAGTTCAGACGTGTGC-3'. The second nucleotide in the sequence should be $\mathrm{A}$, not $\mathrm{C}$, and the correct sequence is 5'-CAAGCAGAAGACGGCATACGAGATXXXXXXGTGACTGGAGTTCAGACGTGTGC-3'.

The error has been corrected in the PDF and HTML versions of this article. 